\title{
Evolución de las poblaciones de insectos en una tabla de arroz de las marismas del bajo Guadalquivir.
}

\author{
E. Cano y A. Jiménez \\ Dpto. de Fisiología y Zoología. Fac. de Biología. Universidad de Sevilla. Avda. Reina Mercedes, 6. 41012 \\ Sevilla.ecano@us.es
}

\section{RESUMEN}

En las marismas del Bajo Guadalquivir el cultivo de arroz se realiza en un flujo continuo de agua. Mediante este sistema se mantiene el agua oxigenada y permanece con temperaturas suaves, entre 20 y $25^{\circ} \mathrm{C}$. Las labores agrícolas realizadas en este cultivo son las tradicionales de la zona. Los individuos capturados pertenecen al Orden Diptera (Fam. Chironomidae, Fam. Ephydridae y otras familias), Orden Coleoptera (Fam. Dytiscidae y Fam. Hydrophilidae), Orden Hemiptera (Fam. Corixidae y Fam. Notonectidae), Orden Odonata (Fam. Libellulidae y Fam. Calopterygidae) y Orden Ephemeroptera (Fam. Ephemeridae). Cuantitativamente los distintos grupos aparecen representados de una forma muy heterogénea, pero en cualquier caso el grupo más representativo es el de los dípteros, y más concretamente los pertenecientes a la familia Chironomidae. Se producen importantes variaciones en la comunidad de insectos de la tabla de arroz, las cuales pueden ser debidas, principalmente, a los tratamientos con pesticidas que se aplican al cultivo, y a las oscilaciones en el nivel del agua de la tabla.

Palabras clave: Insectos, marismas, arrozal.

\begin{abstract}
In the lower Guadalquivir river marshes, rice is grown with a flow-through system, which keeps the water oxygenated and with mild temperatures around $20-25^{\circ} \mathrm{C}$. Traditional farming workings are used in this area. Individuals collected belong to the Order Diptera (Fam. Chironomidae, Fam. Ephydridae and others families), Order Coleoptera (Fam. Dytiscidae and Fam. Hidrophylidae), Order Hemiptera (Fam. Corixidae and Fam. Notonectidae), Order Odonata (Fam. Libellulidae and Fam. Calopterygidae) and Order Ephemeroptera (Fam. Ephemeridae). Quantitatively the different groups are represented in a very heterogeneous way, however, the most numerous are the dipterans, in particular, the Chironomidae. There are important variations in the insect community of a rice field. These variations can be due to pesticide treatments applied to the rice, and water level oscillations.
\end{abstract}

Keywords: Insects, marshes, rice field.

\section{INTRODUCCION}

En las marismas del Bajo Guadalquivir, en la provincia de Sevilla hay destinadas al cultivo de arroz unas 35.500 ha. El periodo de este cultivo abarca desde finales de abril hasta septiembre $u$ octubre (Aguilar, 2001) y las tablas son colonizadas por diversos insectos acuáticos los cuales están normalmente asociados con otros hábitats acuáticos temporales (Usinger, 1956; Zalom, 1981). Debido a que estudios en otros agro-ecosistemas muestran que la abundancia y estructura de la comunidad de los insectos puede variar dependiendo de las labores que se practiquen en el cultivo (Dritschilo \& Wanner, 1980; Madsen \& Madsen, 1982; Hesler et al., 1993), en el presente trabajo hemos seguido la evolución de las poblaciones de insectos a nivel taxonómico de familia de una tablas de arroz (tanto cualitativa como cuantitativamente).

En las marismas del Bajo Guadalquivir el cultivo de arroz se realiza, de forma característica, en un flujo continuo de agua. Mediante este sistema se mantiene el agua oxigenada y permanece con temperaturas suaves, entre 20 y $25{ }^{\circ} \mathrm{C}$. Las labores agrícolas que se practican son las 
tradicionales, es decir, las preparatorias de la tierra para la próxima siembra (fangueo, arado, nivelado...), las de presiembra en las que queda comprendida la de fertilización, inundación de las tablas y siembra del arroz (Oryza sativa, L.) y por último las labores que se realizan durante el desarrollo fenológico del cultivo en el que como norma general se realiza una seca de 7 días y dos aplicaciones de pesticidas (una a la semana de la siembra y otra coincidiendo con la seca).

\section{MATERIAL Y MÉTODOS}

El estudio fue llevado a cabo en el periodo que abarcó el cultivo de arroz en la campaña de 1996 y concretamente desde el 4 de junio al 12 de septiembre, en una tabla de arroz (Fig. 1) situada en la finca de los Hnos. Lara (UTM 29SQB534246), incluida en las marismas del
Bajo Guadalquivir. En total fueron realizados 15 muestreos con una periodicidad semanal. En todos ellos se realizaron tomas de muestras de suelo, con una estructura metálica de sección cuadrangular que delimitaba una superficie de $30 \mathrm{~cm}^{2}$ y la cual era enterrada a $3 \mathrm{~cm}$ de profundidad en 3 puntos de la tabla, como se muestra en la figura 1. Los tres puntos se escogieron teniendo en cuenta las diferencias existentes en el flujo de agua dentro de una tabla de arroz, uno estaba en la entrada de agua, otro casi en el centro de ésta y el último se localizaba en la salida del agua. Las condiciones físico-químicas del agua de la tabla eran medidas en cada muestreo.

Las labores que se realizaron en el cultivo fueron las típicas de la zona. La tabla fue inundada el 4 de Junio a una altura media de aproximadamente $10 \mathrm{~cm}$. El abonado se realizo 3 días antes con Urea- $46 \% \mathrm{~N}$. La siembra se realizó con avioneta el 5 de Junio, para ello se utilizó semilla cer-

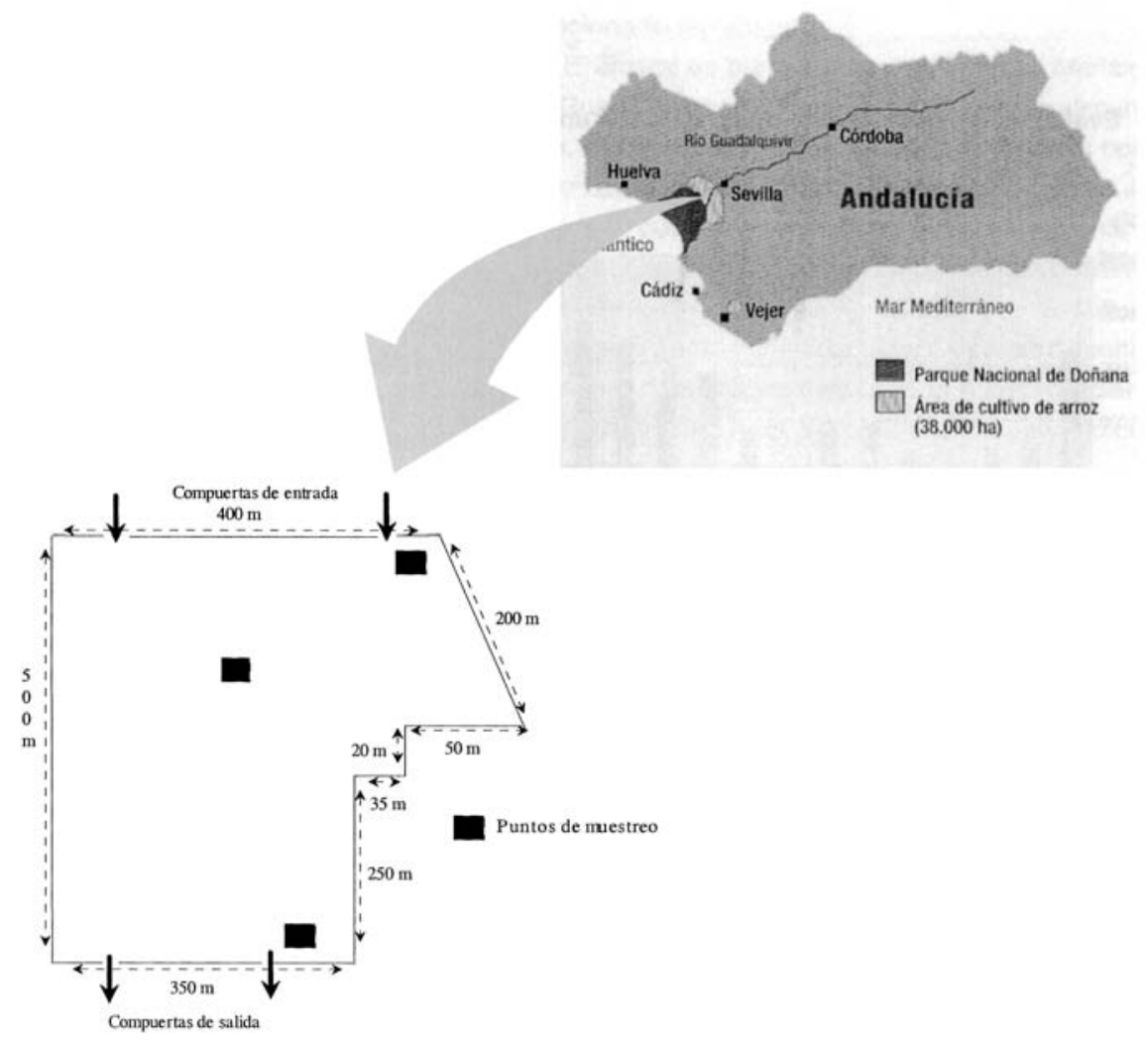

Figura 1. Localización de la zona de estudio. Esquema de la tabla de arroz muestreada. Study site location. Sampled rice field croquis. 
tificada de Oryza sativa var. indica Thaibonet pregerminada. A los 3 días de la siembra el nivel del agua fue descendido a $3 \mathrm{~cm}$ durante 4 días, para facilitar la implantación de la plántula de arroz, y a la vez se le aplicó el insecticida Malatión en una concentración de 1.5 1/ha, para el control de los quironómidos. A los cuarenta días se realizó la seca, que consistió en mantener la tabla sin agua durante 7 días, coincidiendo con la aplicación del herbicida (Bentazona, Sal Sódica, $40 \%$ + MCPA, Sal Amina, $6 \%$, p/v.L.S.) en una concentración de 4 1/ha. A los dos meses se realizo un segundo tratamiento de insecticidas con una mezcla al 50\% de Malatión + Triclorfón y a una concentración de $1.5 \mathrm{l} / \mathrm{ha}+1.5 \mathrm{~kg} / \mathrm{ha}$. El 12 de septiembre la tabla fue secada para una semana mas tarde recoger la cosecha.

Las muestras de suelo recogidas, eran transportadas al laboratorio donde eran lavadas mediante un sistema de 4 tamices apilados y con un diámetro de malla de $3,1,0.3$ y $0.1 \mathrm{~mm}$. Al sedimento resultante del tamizado se le aplicaba la técnica de flotación del azúcar (Anderson, 1959) para la recolección de los individuos más pequeños. Los ejemplares separados eran conservados en alcohol al $70 \%$ para su posterior identificación y conteo.

Los factores físico-químicos medidos a lo largo del periodo de estudio fueron analizados por medio de un análisis de la varianza (ANOVA), a un nivel de significación de 0,05. Para realizar el estudio estadístico de los datos de capturas obtenidos, entre los distintos puntos de muestreo, se les ha aplicado el test de Kruskal-Wallis, a un nivel de significación del $95 \%$, previa comprobación de la no normalidad de los datos.

\section{RESULTADOS Y DISCUSIÓN}

Las variables físico-químicas medidas a lo largo de los distintos muestreos, aparecen reflejados en la Tabla 1. Los individuos capturados pertenecen al Orden Diptera (Fam. Chironomidae, Fam. Ephydridae y otras familias), Orden Coleoptera (Fam. Dytiscidae y Fam. Hidrophylidae), Orden Hemiptera (Fam. Corixidae y Fam. Notonectidae), Orden Odonata (Fam. Libellulidae y Fam. Calopterygidae) y Orden Ephemeroptera (Fam. Ephemeridae). En la Tabla 2 se observa que cuantitativamente las distintas familias aparecen representadas de una forma muy heterogénea y con elevadas fluctuaciones a lo largo del periodo de estudio en el número de individuos capturados de cada grupo. El grupo más representativo es el de los dípteros, y más concretamente los pertenecientes a la familia Chironomidae.

Tabla 1. Variables físico-químicas recogidas en los muestreos de la tabla. Physical-chemical factors collected in the rice field.

\begin{tabular}{|c|c|c|c|c|c|c|c|c|c|c|c|c|c|c|c|c|c|}
\hline & \multicolumn{5}{|c|}{ Junio } & \multicolumn{3}{|c|}{ Julio } & \multicolumn{4}{|c|}{ Agosto } & \multicolumn{3}{|c|}{ Sept. } & \multirow{2}{*}{$\mathbf{x}$} & \multirow{2}{*}{ DS } \\
\hline & 4 & 14 & 21 & 27 & 4 & 10 & 19 & 26 & 2 & 9 & 14 & 23 & 29 & 5 & 12 & & \\
\hline Conduct. (mS) & 2.17 & 3.52 & 2.94 & 0.52 & 0.54 & 0.57 & 0.69 & & & 2.56 & 2.46 & 2.2 & 2.04 & 2.15 & 2.13 & 1.88 & 0.99 \\
\hline Oxígeno (ppm) & 3.8 & 4.8 & 6.7 & 4.5 & 4.6 & 3 & 2 & 4 & 3.3 & 2.5 & 4.6 & 2.6 & 3.2 & 6.1 & 2.4 & 3.87 & 1.2 \\
\hline Nivel agua $(\mathrm{cm})$ & 12 & 6 & 9 & 10 & 12 & 12 & 8 & 19 & 12 & 17 & 15 & 15 & 12 & 14 & 16 & 12.6 & 3.1 \\
\hline $\mathrm{T}^{\mathrm{a}} \operatorname{Max} .\left({ }^{\circ} \mathrm{C}\right)$ aire & 34 & 38 & 30 & 37 & 27 & 36.5 & 41.5 & 30 & 35 & 37 & 32 & 34 & 31.5 & 29 & 27 & 33.3 & 4.27 \\
\hline $\mathrm{T}^{\mathrm{a}} \mathrm{Med}\left({ }^{\circ} \mathrm{C}\right)$ aire & 23 & 27.8 & 23 & 25.2 & 21.5 & 27 & 30.5 & 23.5 & 26.5 & 27.2 & 24 & 23.5 & 23.7 & 23 & 21.5 & 24.7 & 2.56 \\
\hline $\mathrm{T}^{\mathrm{a}}$ Min. $\left({ }^{\circ} \mathrm{C}\right)$ aire & 12 & 17.7 & 16 & 13.5 & 16 & 17.5 & 19.5 & 17 & 18 & 17.5 & 16 & 13 & 16 & 17 & 16 & 16.2 & 2.01 \\
\hline $\mathrm{T}^{\mathrm{a}}$ agua $\left({ }^{\circ} \mathrm{C}\right)$ & 22.9 & 22.6 & 20 & 23.6 & 24.3 & 22.7 & 24 & 21.7 & 21 & 22.4 & 20.6 & 19.2 & 21.3 & 20.6 & 19.3 & 21.7 & 1.8 \\
\hline $\mathrm{pH}$ & 8 & 8 & 8.2 & 7.5 & 8.2 & 7.5 & 7.5 & 7.9 & 7.9 & 7.6 & 8 & 7.6 & 7.6 & 7.7 & 7.7 & 7.79 & 0.19 \\
\hline
\end{tabular}


Tabla 2. Ejemplares obtenidos en los puntos de muestreo durante el cultivo de arroz. (E: entrada; I: interior; S: salida). Individuals collected in the sampling points during rice growing. (E: input; I: interior; S: output).

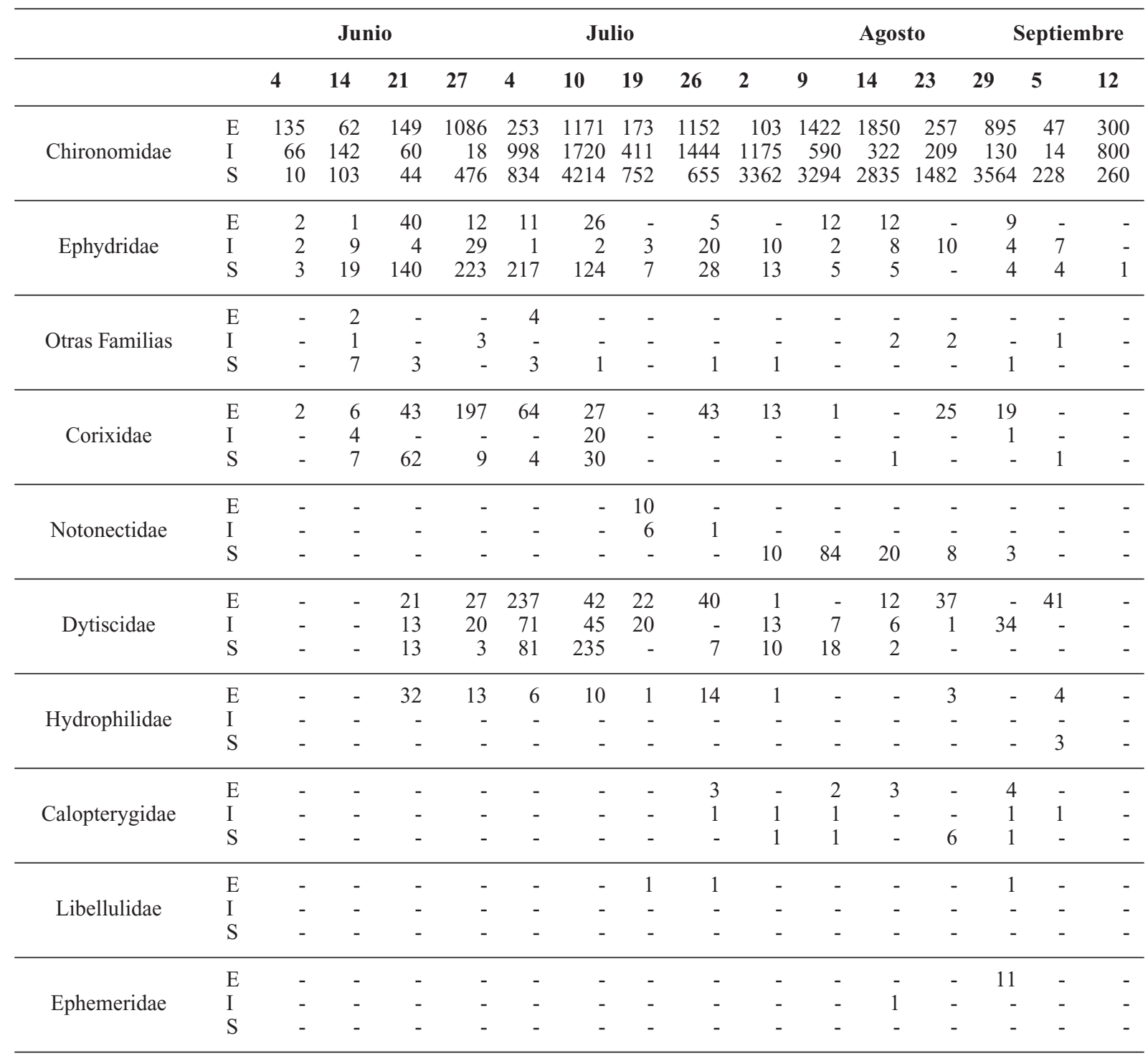

El cultivo de arroz en las marismas del Bajo Guadalquivir presenta como característica peculiar, el que se realiza en un flujo continuo de agua, sistema mediante el cual se pretende mantener el agua oxigenada y no permitir una subida excesiva de la temperatura del agua cuando las temperaturas ambientales lo hacen, hecho que podemos comprobar en los resultados obtenidos en la Tabla 1. El agua utilizada en este cultivo procede del río Guadalquivir el cual en esta zona (50 a $70 \mathrm{~km}$. de la desembocadura) presenta grandes variaciones en sus características químicas. Debido a que el tramo final del río Guadalquivir, entre Sevilla y su desembocadura, presenta un ligero desnivel, estando sometido al régimen de mareas. El denominado "tapón salino" (zona de transición entre agua salada del mar y dulce del río) puede ser desplazado hacia el sur mediante desembalses de una presa (en Alcalá del Río, Sevilla) situada a unos 
$100 \mathrm{~km}$. de la desembocadura del río (Aguilar, 2001). Estas variaciones se observan sobre todo en la conductividad, donde a lo largo del periodo de muestreo presenta valores $(0.52-3.52 \mathrm{mS})$ con diferencias significativas. Los valores de $\mathrm{pH}$ que se obtienen son los normales de este cultivo, entre 7.5 y 8.2. Los valores del oxígeno disuelto, a lo largo del periodo de estudio, no presentan diferencias significativas.

Las alteraciones en la dinámica y estructura de la comunidad de invertebrados acuáticos en las tablas de arroz por el uso de pesticidas (Malatión y Triclorfón), podría tener importantes consecuencias agro-ecológicas (Simpson et al., 1994). Las variaciones que se observan en la comunidad acuática de nuestra tabla de arroz, pueden estar relacionadas, principalmente con los tratamientos de pesticidas, aunque también pueden estarlo con las variaciones en el nivel del agua de la tabla. Durante los diez o doce primeros días del cultivo los niveles poblacionales de los distintos grupos presentes son muy bajos ya que en este periodo se realiza la bajada del nivel del agua y la aplicación de Malatión para el control de quironómidos (Fig. 2). A partir de este momento comienza un aumento de estos niveles poblacionales para todos los grupos, debido a que la actividad del Malatión ha descendido puesto que la vida media de este insecticida aplicado directamente en agua ha sido estimada en 3 días con altos niveles de
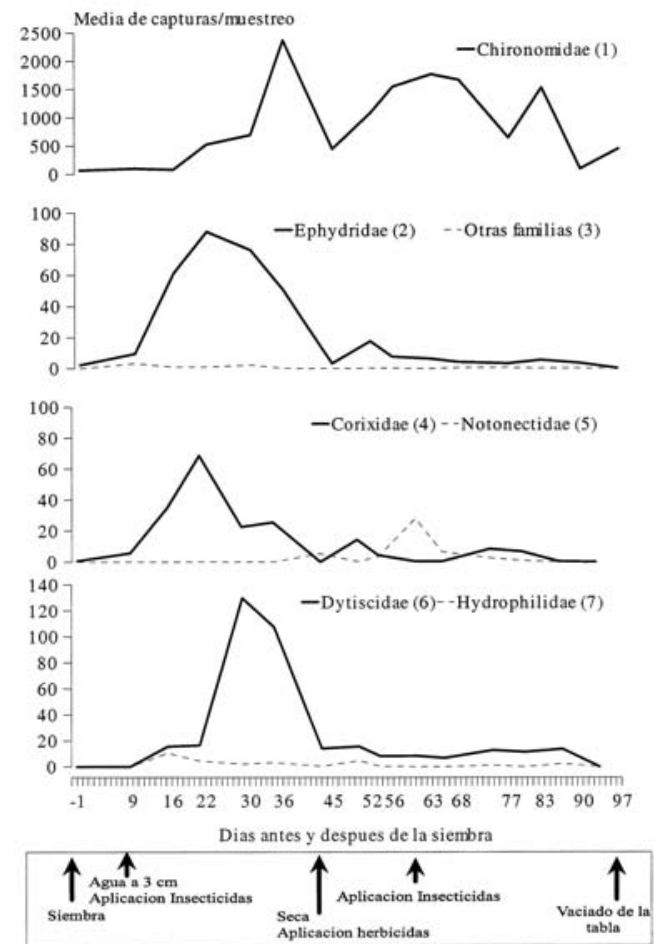

\begin{tabular}{|c|c|c|c|c|c|c|c|c|c|c|c|c|c|c|c|}
\hline \multicolumn{16}{|c|}{ Capturas medias/muestreo \pm DS } \\
\hline (1) & $70.3 \pm$ & $102.3 \pm$ & $84.3 \pm$ & $526 \pm$ & $695 \pm$ & $2368.3 \pm$ & $445.3 \pm$ & $1083 \pm$ & $1546.7 \pm$ & $1768.7 \pm$ & $1669 \pm$ & $649.3 \pm$ & $1529.7 \pm 1$ & $96.3 \pm$ & $453.3 \pm$ \\
\hline \multirow{3}{*}{ (2) } & 62.6 & 40 & 56.6 & 535 & 391.5 & 1621.8 & 291 & 398.9 & 1661 & 1384.9 & 1266.2 & 721.5 & 802.8 & 115.2 & 300.9 \\
\hline & $2.3 \pm$ & $9.6 \pm$ & $61.3 \pm$ & $88 \pm$ & $76.3 \pm$ & $50.6 \pm$ & $3.3 \pm$ & $17.6 \pm$ & $7.6 \pm$ & $6.3 \pm$ & $8.3 \pm$ & $3.3 t$ & $5.6 \pm$ & $3.6 \pm$ & $0.3 \pm$ \\
\hline & 0.57 & 9.02 & 70.46 & 117.2 & 121.9 & & 3.51 & 11.67 & 6.81 & 5.13 & 3.51 & 5.77 & 2.88 & 3.51 & 0.57 \\
\hline \multirow[t]{2}{*}{ (3) } & $0 \pm 0$ & $3.3 \pm$ & $1 \pm$ & $1 \pm$ & $2.3 \pm$ & 0 & $0 \pm 0$ & $0.3 \pm$ & $0.3 \pm$ & $0 \pm 0$ & $0.6 \pm$ & $0.6 \pm$ & $0.3 \pm$ & $0.3 \pm$ & $0 \pm 0$ \\
\hline & & 3.21 & 1.73 & 1.73 & 2.08 & 0.58 & & 0.58 & 0.58 & & 1.15 & 1.15 & 0.5 & 0.58 & \\
\hline \multirow[t]{2}{*}{ (4) } & $0.6 \pm$ & $5.6 \pm$ & $35 \pm$ & $68.6 \pm$ & $22.6 \pm$ & $25.6 \pm$ & $0 \pm 0$ & $14.3 \pm$ & $4.3 \pm$ & $0.3 \pm$ & $0.3 \pm$ & $8.3 \pm$ & $6.6 \pm$ & $0.3 \pm$ & $0 \pm 0$ \\
\hline & 1.15 & 1.52 & 31.76 & 111.23 & 35.85 & 5.13 & & 24.8 & & & 0.5 & & 10.69 & & \\
\hline (5) & $0 \pm 0$ & $0 \pm 0$ & $0 \pm 0$ & $0 \pm 0$ & $0 \pm 0$ & $0 \pm 0$ & $\begin{array}{l}5.3 \pm \\
5.03\end{array}$ & $\begin{array}{l}0.3 \pm \\
0.58\end{array}$ & $\begin{array}{l}3.3 t \\
577\end{array}$ & $\begin{array}{l}28 \pm \\
4.49\end{array}$ & $6.6 \pm$ & $\begin{array}{l}2.6 \pm \\
4.62\end{array}$ & $1 \pm$ & $0 \pm 0$ & $0 \pm 0$ \\
\hline \multirow[t]{2}{*}{ (6) } & $0 \pm 0$ & $0 \pm 0$ & $15.6 \pm$ & $16.6 \pm$ & $129.6 \pm$ & $107.3 \pm$ & $14 \pm$ & $15.6 \pm$ & $8 \pm$ & $8.4 \pm$ & $6.6 \pm$ & $\frac{4.02}{12.6 \pm}$ & $11.3 \pm$ & $13.6 \pm$ & $0 \pm 0$ \\
\hline & & & 4.62 & 12.34 & 93.08 & 110.57 & 12.16 & 21.36 & 6.24 & & & 21.08 & 19.63 & 23.67 & \\
\hline (7) & $0 \pm 0$ & $0 \pm 0$ & $\begin{array}{l}10.6 \pm \\
18.47\end{array}$ & $\begin{array}{c}4.3 \pm \\
7.5\end{array}$ & $\begin{array}{c}2 t \\
346\end{array}$ & $\begin{array}{l}3.3 \pm \\
5.77\end{array}$ & $\begin{array}{l}0.3 t \\
0.58\end{array}$ & $\begin{array}{l}4.6 \pm \\
8.08\end{array}$ & $\begin{array}{l}0.3 \pm \\
0.58\end{array}$ & $0 \pm 0$ & $0 \pm 0$ & $\begin{array}{c}1 \pm \\
1.73\end{array}$ & $0 \pm 0$ & $\begin{array}{l}2.3 \pm \\
2.08\end{array}$ & $0 \pm 0$ \\
\hline
\end{tabular}

Figura 2. Evolución de las familias de insectos (capturas medias/muestreo) encontradas en la tabla de arroz. Evolution of the insect families (mean values/sampling) in the rice field. 
actividad, y con un descenso hasta cero a los 7 días del tratamiento (Stevens, 1991). También en esta fecha el nivel del agua es aumentado hasta unos $10 \mathrm{~cm}$, con lo cual se diluye el pesticida y se produce una entrada de organismos en el sistema, por la compuerta de entrada.

En el caso de los quironómidos (Fig. 2) el aumento de la población comienza más tarde debido a que viven en el sedimento; no son introducidos con el nuevo agua y por lo tanto, necesitan colonizar de nuevo el medio inundado con puestas. Para que aparezcan nuevamente larvas, en nuestra zona se necesitan unos 2 días, tiempo que tardan en eclosionar los huevos (Aguilar, 2001). En la figura 2 vemos que la curva comienza a subir posteriormente, ésto se debe a que el estudio se realizó contabilizando larvas en estadios L3 y L4, y éstos se consiguen en unos 4 u 8 días, tras la eclosión (Aguilar, 2001; Stevens, 1993).

Tras este aumento de las poblaciones, éstas llegan a un máximo poblacional y luego comienza un descenso, del cual no se recuperan ya que por esas fechas se produce la seca y la aplicación de herbicidas (Fig. 2). En el caso de los quironómidos vemos unos nuevos picos, que son debidos a su carácter oportunista, capaz de colonizar nuevamente la tabla de arroz tras ser llenada (Cantrel \& McLachlan, 1977). El segundo aumento de esta población se ve afectado por la aplicación de un segundo tratamiento de insecticidas (Malatión+Triclorfon).

Se observan diferencias en las capturas de ejemplares de las familias más numerosas encontradas en este estudio entre los distintos puntos muestreados. Éstas no son significativas estadísticamente para quironómidos $(\mathrm{p}=$ $0.275)$, efídridos $(\mathrm{p}=0.208)$, ditíscidos $(\mathrm{p}=$ $0.422)$, aunque sí en coríxidos $(\mathrm{p}=0.003)$. A pesar de no presentar diferencias estadísticas, en los dos primeros grupos se aprecia un mayor número de ejemplares en el punto de salida del agua, lo que nos indicaría que existe un arrastre hacia este lugar. Sólo se aprecia en éstas familias ya que son las que tienen larvas poco activas y fáciles de arrastrar. En los coríxidos se observa que el mayor número de ejemplares se encuentra en la entrada. Las larvas de los ditíscidos son muy activas y nadan con facilidad por los arrozales, por ello las medias de capturas en los distintos puntos son muy similares.

\section{BIBLIOGRAFÍA}

AGUILAR, M. 2001. Cultivo del arroz en el Sur de España. Ed. Caja de Ahorros el Monte. 189 pp.

ANDERSON, R. O. 1959. A modified flotation technique for sorting bottom faune samples. Limnol. Oceanogr., 4: 223-225.

CANTREL, M. A. \& A.J. McLACHLAN. 1977. Competition and chironomid distribution patterns in a newly flooded lake. Oikos, 29: 429-433.

DRITSCHILO, W. \& D. WANNER. 1980. Ground beetle abundance in organic and conventional corn fields. Environmental Entomology, 9: 629-631.

GRIGARICK, A. A. 1984. Problems with rice invertebrates and their control in the United States. Prot. Ecol., 7: 105-114.

HESLER, L. S., A. A. GRIGARICK, J. O. MICHAEL \& A. T. PALRANG. 1993. Arthropod Fauna of Conventional and Organic Rice Fields in California. J. Econ. Entomol., 86 (1): 149-158.

MADSEN, H. F. \& B. J. MADSEN. 1982. Populations of beneficial and pest artrophods in an organic and a pesticide-treated apple orchard in British Columbia. Can. Entomol., 114: 1083-1088.

SIMPSON, I. A., P. A. ROCER, R. OFICIAL. \& I. F. GRANT. 1994. Effects of nitrogen fertilizer and pesticide management on floodwater ecology in a wetland ricefields. II. Dynamic of microcrustaceans and dipteran larvae. Biological Fertil Soils, 17: 138-146.

STEVENS, M. M. 1991. Insecticide treatements used against a rice bloodworm, Chironomus tepperi Skuse (Diptera: Chironomidae): toxicity and residual effects in water. J. Econ. Entomol., 84: 795-800.

STEVENS, M. M. 1993. Larval development in Chironomus tepperi Skuse (Diptera: Chironomidae) under laboratory conditions. Env. Entomol., 22: 776780.

USINGER, R. L. 1956. Aquatic Hemiptera. In: Aquatic Insects of California. R. L. Usinger (ed.). 182-228. University of California Press, Berkeley.

ZALOM, F. G. 1981. Interactions potentially affecting the seasonal abundance of selected aquatic invertebates in a rice-field habitat. Hydrobiologia, 80: 251255. 